\title{
Looking Through Paintings
}

\author{
Joris $\mathrm{Dik}^{1}$
}

1. Materials Science and Engineering, Delft University of Technology. Delft, The Netherlands

Paintings are windows into the history of human kind, our history. None of us can claim never to have set foot in a museum to appreciate these beautiful works of art. The magnificent brushwork of Velazquez, the vivid colors and light effects of el Greco, the fine and detailed work of Rembrandt; and the emotional honesty and bold colors of Van Gogh are characteristics to be admired. They are all indications of the time, place and specific cultural moment in which the artists lived. Historians have been using this information in combination with correspondence and narrative accounts to reconstruct the moments of history in which the works came to light.

For hundreds of years the public has only seen the surface of famous masterpieces by artists such as Van Gogh and Rembrandt. Now, through a melding of innovative scientific techniques and art connoisseurship, completely different paintings and important clues about the artists themselves can be uncovered one layer at a time using non-invasive technologies. Researchers can calculate how and when an artist first began working on their composition by tracing fingerprints, brushstrokes and analyzing different layers of paint pigments. Art historians are increasingly turning to particle physics to authenticate masterpieces by artists like Rembrandt and Van Gogh, as well as to explore mysterious artworks lying beneath surface paintings.

Just microns below their paint surface lay a wealth of information on Old Master Paintings. Hidden paint layers can include underdrawing, underpainting or compositional alterations by the painter, or even younger layers related to later restorations. All too often artists simply re-used their canvases and painted a new composition on top. Thus, a look through the paint layer provides a look over the painter's shoulder. These elements, once fully uncovered, sometimes reveal completely different works of art underneath the surface that were abandoned by these great artists and painted over.

Nondestructive imaging of hidden paint layers is usually realized by means of tube-based X-ray radiation transmission radiography (XRR). The absorption contrast in these images is mostly caused by the heavy metal components of pigments employed, such as lead in lead white or mercury in vermillion. Conventional XRR, however, has a number of important limitations. First of all, the observed X-ray absorbance is a summation of all element-specific absorbancies, which reduces the contrast of weakly absorbing elements. Second, lead white primer raises the overall background of the absorption image derived from the paint layers. Finally, the polychromatic character of an X-ray tube further reduces the contrast in radiographic images. As a result, conventional XRR imaging of paintings frequently provides only a fragmentary view of their substructure, which can severely hamper the readability of hidden compositions [1]. In addition to using the absorption of primary X-rays as an imaging method, one can also record the intensity of secondary radiation, emitted by the atoms in the painting while a pencil beam of energetic X-rays is scanned over the surface. This X-ray radiation fluorescence (XRF) technique has the added advantage that the emitted X-ray radiation is element specific. The covering surface layers will not significantly attenuate the high-energy fluorescence signals from heavy elements in the hidden layers; in this manner, the distribution of both minor and major components in the painting can be visualized. The use of high intensity X-ray beams, coupled with high count rate detectors, reduces the 
dwell time for data acquisition to such an extent that large, decimeter sized areas can be scanned in a reasonable time frame. In order to learn about the chemical binding of a specific element, X-ray absorption near edge structure (XANES) measurements can be performed at either the element's K- or L-edge at the most interesting points of the hidden layer.

The application of this technology to work "Patch of Grass" by Van Gogh has revealed the head of a woman in a hidden paint layer [2]. We succeeded in visualizing the hidden face with unprecedented detail. In particular, the distribution of $\mathrm{Hg}$ and $\mathrm{Sb}$ in the red and light tones, respectively, enabled an approximate color reconstruction of the flesh tones. This reconstruction proved to be the missing link for the comparison of the hidden face with Van Gogh's known paintings.

The research into the painting "Old Man with Beard" to determine its attribution determined that the small panel was painted by Rembrandt around 1630, at the end of his time in Leiden. In addition, a selfportrait was revealed when the painting was scanned at the European Synchrotron Radiation Facility (ESRF) in Grenoble and at the Brookhaven National Laboratory (BNL) in New York using Macroscanning X-Ray Fluorescence spectrometry (MA-XRF). The presence of the self-portrait of Rembrandt aided the authentication process [3].

These microscopy and microanalysis techniques have also found a niche in the art conservation field. Over the past years a number of studies have described the instability of the pigment cadmium yellow $(\mathrm{CdS})$. We have shown how cadmium sulfide on paintings by James Ensor oxidizes to $\mathrm{CdSO}_{4} \cdot \mathrm{H}_{2} \mathrm{O}$ [4]. The degradation in the painting "Flowers in a blue vase" by Vincent van Gogh was initially caused by oxidation of the original CdS pigment, similar as Ensor's paintings. However, additional degradation occurs due to the presence of an overlying varnish. The resulting opaque anglesite compound in the varnish, in combination with the underlying $\mathrm{CdC}_{2} \mathrm{O}_{4}$ layer at the paint/varnish interface, account for the orange-gray crust that is disfiguring the painting on a macroscopic level [5].

This presentation will show past successes, present developments and the future potential of these techniques in the realm of art conservation, preservation, authentication and discovery.

\section{References:}

[1] Krug, K., et al., Appl. Phys. A: Mater. Sci. Process. 83 (2006) p 247.

[2] Dik, J., et al., Anal. Chem. 80 (2008) p 6436.

[3] Altfeld, M., et al., Applied Physics A, 111 (2013) p 157.

[4] Van der Snickt G., et al., Anal. Chem. 81 (2009) p 2600.

[5] Van der Snickt G., et al., Anal. Chem. 84 (2012) p 10221. 